\title{
Multilinguales
}

\section{Processus de la néologie dans « Tranche de vie » du Quotidien d'Oran : l'affixation}

The Neology Process in «Tranche de Vie » of Le Quotidien D'oran: The Affixation

\section{Samira Allam-Iddou}

\section{(2) OpenEdition \\ 1 Journals}

Édition électronique

URL : http://journals.openedition.org/multilinguales/987

DOI : $10.4000 /$ multilinguales. 987

ISSN : 2335-1853

Éditeur

Université Abderrahmane Mira - Bejaia

Référence électronique

Samira Allam-Iddou, «Processus de la néologie dans «Tranche de vie » du Quotidien d'Oran :

l'affixation », Multilinguales [En ligne], 6 | 2015, mis en ligne le 01 décembre 2015, consulté le 17 septembre 2019. URL : http://journals.openedition.org/multilinguales/987 ; DOI : 10.4000/ multilinguales.987

Ce document a été généré automatiquement le 17 septembre 2019.

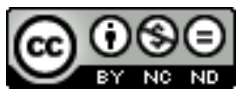

Multilinguales est mise à disposition selon les termes de la Licence Creative Commons Attribution Pas d'Utilisation Commerciale - Pas de Modification 4.0 International 


\title{
Processus de la néologie dans «Tranche de vie » du Quotidien d'Oran : l'affixation
}

The Neology Process in «Tranche de Vie » of Le Quotidien D'oran: The Affixation

\author{
Samira Allam-Iddou
}

1 Par le biais de cette étude sur la créativité lexicale dans les chroniques «Tranche de vie » du journal Le Quotidien d'Oran des années 2009 à 2014, nous tenterons de dégager les procédés de formation les plus productifs de l'affixation et d'en étudier le processus de création à travers les cas les plus significatifs. Nous nous sommes intéressée à la presse écrite francophone parce que, d'emblée, elle paraît un foyer très actif du changement linguistique, par son rapport étroit avec la réalité du terrain. Selon Sader Feghali (2005: 525), «les médias sont le bassin privilégié pour la diffusion des créations néologiques qui témoignent de l'apparition d'une nouveauté ou qui répondent à un besoin d'expression et de communication ".

Des mots, de nouvelles constructions linguistiques, de nouvelles lexies vont délivrer un sens nouveau dicté par des réalités nouvelles.

\section{Notion de néologie journalistique et données préliminaires}

2 S'agissant de l'étude globale de tous les procédés de néologie à l'œuvre dans les chroniques de 2009 à 2014, la constitution du corpus de néologismes n'a pas été chose aisée pour plusieurs raisons. Parmi elles, citons la première qui tenait à la sélection de l'unité en fonction de sa pertinence dans le cadre de notre problématique ; la seconde, à la détermination d'un seuil temporel significatif de nouveauté étant donné sa variabilité; la troisième, à l'estimation de la nouveauté dans les circonstances d'interlocution; la quatrième à la détection d'un nouveau sens inhérent à ces innovations linguistiques. Quant à la collecte proprement dite, au dépouillement 
systématique de revues ou d'ouvrages spécialisés dans un domaine particulier, nous avons préféré établir un corpus large en puisant dans des sources journalistiques francophones d'informations générales, à savoir Le Quotidien d'Oran et, plus spécialement, la chronique intitulée Tranche de vie signée du sobriquet «El-Guellil »1, tenue par le journaliste Baba Ahmed Fodil.

3 La chronique, type de discours journalistique considéré comme propice à la création lexicale, est marquée par sa façon spécifique d'aborder des sujets très divers, émanant de la réalité sociale. Sa définition, telle qu'élaborée par Jean-Claude Picard (1999: 38), permet de mettre en lumière les caractéristiques favorables à la création lexicale :

La chronique est un genre journalistique dont il est périlleux de proposer une définition tellement elle apparaît comme une sorte de fourre-tout qui, peu importe le sujet traité, se déploie au gré des humeurs et des opinions de son auteur. D'une chronique à l'autre et d'un chroniqueur à l'autre, il est bien difficile de dégager des éléments communs... si ce n'est la totale liberté d'expression dont jouit son auteur. Une liberté qui s'exprime autant dans le choix des sujets et la teneur des propos que dans le style d'écriture. ${ }^{2}$

«Fourre-tout » quant aux domaines dont relèvent les sujets traités, « au gré des humeurs et des opinions» quant à la façon dont ces sujets sont choisis, "totale liberté ...dans la teneur des propos et le style d'écriture» quant à leur tonalité. Notre chronique est donc tout à fait désignée pour constituer un corpus d'étude de la créativité lexicale. Nous avons particulièrement porté une attention sur les chroniques du mois de janvier 2009 au mois de juin 2014 parce que c'est une période riche en événements nationaux et internationaux sur le plan politique, économique, sportif et culturel.

Le repérage des phénomènes néologiques s'est volontairement effectué manuellement ; la collecte automatique, selon Sablayrolles (2002: 99), « engendre des erreurs par excès qui lui sont propres, et aussi des erreurs par défaut, dont elle n'a pas l'apanage mais qui ont des conséquences plus graves puisque ces omissions ne sont pas récupérables automatiquement ".

La collecte des données a abouti à l'établissement d'un corpus d'exclusion ${ }^{3}$ : une liste d'unités lexicales non reconnues par le dictionnaire électronique et que nous avons considérées, de ce fait, comme des néologismes.

Notre corpus, dans sa globalité, se compose de 925 unités néologiques dont certaines sont déjà connues car utilisées dans le langage courant et ont fait l'objet de nombreuses recherches universitaires ${ }^{4}$, et d'autres, dans lesquelles nous puiserons nos exemples pour les nécessités de cet article, le sont moins et relèvent en général des idiosyncrasies $\mathrm{du}$ chroniqueur; d'où l'importance que requiert leur contextualisation pour leur interprétation. Les unités néologiques recueillies se distribuent essentiellement en fonction des matrices lexicogéniques internes et externes, selon la terminologie de J.F. Sablayrolles ${ }^{5}$. Elles sont construites selon différents procédés linguistiques (voir tableau en annexe 2).

De prime abord, nous avons constaté que, dans notre corpus, la distribution des unités en fonction des matrices internes ou externes est systématique. En outre, les matrices internes sont prédominantes et semblent incontournables en matière de création lexicale, avec un taux de 82,03\%. En comparaison, les matrices externes représentent la faible proportion de $17,94 \%$.

Les types de matrices néologiques internes composés de :

- la matrice morphosémantique

- la matrice syntaxico-sémantique

- la matrice morphologique

- la matrice pragmatico-sémantique 
Les types de matrices néologiques externes sont principalement constitués des procédés relevant de l'interférence :

- l'emprunt lexical

- le calque

- l'alternance codique

Etant donné l'espace qui nous est imparti pour cet article, nous n'aborderons que la première matrice, la matrice morphosémantique et dans un seul de ses aspects: l'affixation ou dérivation morphologique qui se décline en trois procédés: la préfixation, la suffixation et la formation parasynthétique.

\section{La matrice (interne) morphosémantique : l'affixation ou dérivation morphologique}

4 La création morphosémantique joue un rôle prépondérant dans le lexique journalistique. Notre corpus global compte 489 lexies $(52,86 \%)$ de ce type. Le nombre de procédés de création morphosémantique qui traitent de la substance relationnelle des morphèmes est, certes, important, mais ils sont inégalement distribués. Dans ce qui suit, nous n'examinerons ici le procédé de création par affixation en essayant d'interpréter sa dynamique lexicale. Ce procédé se compose de la préfixation, de la suffixation et de la formation parasynthétique.

D’après les résultats que nous avons enregistrés, ce procédé n'est pas majoritairement employé dans notre corpus. Nous en déduisons que ce procédé n'est pas productif quant à la création de nouvelles lexies : l'affixation ne représente que 76 lexies sur 925, soit un pourcentage de $08,11 \%$. Nous esquisserons, à la fin de l'analyse, une explication pour ce peu d'engouement journalistique pour la néologie par affixation.

- La préfixation est l'opération de création lexicale qui consiste à ajouter un affixe (morphème non -autonome) avant le radical ou le lexème pour former une lexie ${ }^{6}$.

D'après les données recueillies, le nombre de lexies néologiques qui relèvent de la préfixation représente une proportion infime par rapport à la totalité des procédés de notre corpus. Citons un exemple de lexie idiosyncrasique par préfixation pour en comprendre l'élaboration et le fonctionnement : «invrais, infaux » :

Tableau I - préfixation

\begin{tabular}{|l|l|l|}
\hline Préfixe & Néologismes & Illustration \\
\hline in & $\begin{array}{l}\text { "les infaux et } \\
\text { les invrais » }\end{array}$ & $\begin{array}{l}\text { " Revenons donc à nos programmes nationaux de la télé publique qui se déchaîne } \\
\text { invrais, à une heure de grande écoute, au moment où les uns sont branchés sur les } \\
\text { chaînes étrangères, sur leur partie de dominos ou de belote, et les autres avec } \\
\text { moulana, moi non, je reste patriote et je me tape «la syrie » avant le nini, le } \\
\text { mousselssel dar oum dar oum, je ne sais qui » (chronique du 21/08/2011). }\end{array}$ \\
\hline
\end{tabular}




\begin{tabular}{|c|c|}
\hline «l'infaux» & $\begin{array}{l}\text { («l'infaux »): } \\
\text { "(...) Les journaux se décident enfin à faire front commun.(...) C'est ainsi qu'ils ont } \\
\text { décidé, à la veille des échéances que vous connaissez de ne plus faire dans tachrak } \\
\text { el foum. Plus d'opposition. Plus de commentaires acerbes. Plus d'analyse qui } \\
\text { dérange. Plus de billets provocateurs. Plus de débat contradictoire. Oualou. Que } \\
\text { des infos officielles. Des couvertures officielles. Des photos d'agence officielle. Des } \\
\text { verbes officiels.(...) C'est qu'ils ne sont pas dupes les éditeurs. Ils savent que } \\
\text { l'opposition qu'ils affichent, à travers les écrits publiés sur leurs journaux, sert } \\
\text { plus leurs opposants. C'est pour cela qu'ils ont décidé, dorénavant, de ne publier } \\
\text { que les articles dans le sens du "poilitique ».(..) Et nous refusons de vous servir } \\
\text { de caution. Ceci est de la fiction, bien sûr, c'est de l'infaux! (chronique du } \\
25 / 02 / 2009 \text { et du 04/06/2011). }\end{array}$ \\
\hline
\end{tabular}

5 A première vue, les dérivés négatifs relevés répondent au schéma de dérivation courant: « préfixes dé / in + substantif / adjectif ». Mais, l'effet discursif de la négation lexicale de ces préfixés n'est pas identique à celui de la négation syntaxique avec «ne... pas» (ne pas être "vrai » / "faux»). Dans la chronique journalistique, en général et telle que définie plus haut, le chroniqueur, à l'assertion catégorique qui exclut toute adresse à l'autre, au lecteur, préfère créer un mot de façon à affirmer la même chose mais en faisant de l'autre (le lecteur) son témoin, voire son complice.

A ce propos, citons les deux lexies contigües «les infaux» et «les invrais». A première vue, il semble qu'elles soient toutes deux formées du préfixe privatif in- et de l'adjectif/ substantif -faux/ -vrai. Mais elles sont situées dans un contexte qui en oriente l'interprétation: " programmes nationaux de la télé publique qui se déchaîne en quatre mêmes chaînes (...) à une heure de grande écoute (...)»; il s'agit donc du journal télévisé et de ce fait « infaux» laisse entendre «info(s) », diminutif courant pour « information (s) ». De plus l'association d'« infaux» à « info(s) » est familière pour le lecteur de «Tranche de vie ».

En effet, «l'infaux » paraît dans une chronique reprise deux fois en amont: en février 2009 et en juin 2011. Ainsi, dans le contexte de l'information journalistique de cette première chronique (voir citation supra), la lexie « infaux », substantivée, se superpose, par association, au substantif «info(s) ». L'équivalence phonique des deux substantifs induit, par contamination, une association sémantique: «infos» / "infaux»: les informations sont de "l'info" (informations réelles) mais dans le sens «faux» de «l'infaux». La lexie "l'infaux» insérée dans la formule «c'est de l'infaux» se superpose à l'expression médiatique lexicalisée, "info ou intox", pour associer « infaux » à " intox » dans le langage idiosyncrasique du chroniqueur.

Le lecteur de "Tranche de vie» a donc déjà rencontré par deux fois cette lexie (infaux »), dans le contexte évoqué ci-dessus, avant la troisième où elle apparaît (deux mois après la seconde), au pluriel, dans un contexte quasi identique (informations journalistiques télévisuelles), accompagnée de celle de «les invrais » concernée par la préfixation privative et à laquelle elle est coordonnée. "Les infaux» et "les invrais » sont donc de même nature et forment (mais sur un plan syntagmatique) une paire corrélative dans laquelle « faux » se superpose à « vrais ».

Pour un lecteur non familier de cette chronique, les deux lexies, construites sur le même modèle de préfixation, s'opposent comme le "faux " au "vrai »: "les in-faux" (littéralement : qui ne sont pas faux) sont vraies et «les in-vrais " (littéralement : qui ne sont pas vrais) sont faux, s'agissant des journaux télévisés. 
Mais un lecteur fidèle de cette chronique journalistique aura tendance à restituer à « (les) infaux", par association sémantico-prosodique, la mémoire de "(l')infaux" («faux »/« intox »). Ce qui a pour effet de rendre «(les) infaux », en tant qu'opposé à " (les) invrais », suspect quant à la crédibilité de ce qui n'est pas faux : «l'infaux »/ «l'info » étant «de l'infaux» / "de l'intox», «les infaux», également associés à "l'intox», sont aussi «les invrais» sur les "quatre mêmes chaînes (...) à une heure de grande écoute »: de vraies informations mais fausses ou de fausses informations mais vraies. D'où l'intérêt pour le chroniqueur de recourir au préfixe privatif plutôt qu'à la formule de négation «ne ...pas »: il dit à la fois ce qui est (l'information se donne comme vraie) et ce qui n'est pas (l'information n'est pas vraie) : de vraies fausses informations.

Donc, nous pouvons même dire que la création lexicale par préfixation modifie la valeur sémantique des mots, du moins pour les dérivés négatifs. Pour tenter d'expliquer sa très faible proportion dans "Tranche de vie ", nous pouvons souligner que ce processus de préfixation est complexe et nécessite une participation active du lecteur pour l'interprétation du message délivré par les lexies; le style de cette chronique étant plutôt ludique.

- La suffixation est une opération de création lexicale qui consiste à ajouter un affixe (un suffixe flexionnel ou dérivationnel) au radical pour former une lexie. Le procédé est très répandu dans la chronique «Tranche de vie » et les suffixes recensés sont des suffixes de dérivation essentiellement nominale ${ }^{7}$ :-(a)tion, -iste -isme, -eur, -esque, -asse -asser, -esse, -erie, age, ard, etc. Leur adjonction confère une nouvelle valeur sémantique aux néologismes obtenus. Le chroniqueur use, certes des néologismes entrés dans le langage courant ( " hitiste » ou « barreaudage », etc.), mais en crée aussi de nouveaux sur le même modèle. Le radical peut être de langue arabe, de langue française ou d'une autre langue, comme l'anglais par exemple ; le critère de sélection du radical étant son usage en langage courant tel trabendo de «trabendiste», qui existe déjà dans le langage populaire, ou parking de « parkingueur ", créé par le chroniqueur, etc. La lexie peut être composée d'un mot ou plus, ou encore de toute une expression (voir infra). Voici quelques exemples regroupés selon leur coprésence dans une même chronique ou dans des chroniques différentes mais traitant d'un même thème :

Tableau II - Suffixation

\begin{tabular}{|c|c|c|}
\hline Suffixes & Néologismes & Illustration \\
\hline $\begin{array}{l}\mathrm{N}^{\circ} 1 . \\
-(a) \text { tion } \\
-e r\end{array}$ & $\begin{array}{l}\text { Buveresse } \\
\text { (bourrécole) } \\
\left(l^{\prime}\right) \text { Ânation } \\
\text { bourricoler }\end{array}$ & $\begin{array}{l}\text { "On ne sait pas conduire. (...)C'est quoi donc la solution? (...) L'Ane. On se } \\
\text { déplacera à dos d'âne. Comme ça, au moins, plus besoin de parking. Juste des } \\
\text { kouris pour bourricots. Toutes nos villes sont bourrées de cités qui n'ont rien à } \\
\text { envier aux kouris d'antan. (...)Plus de retrait de permis pour conduite en état de } \\
\text { buveresse. (...) Ainsi, on commencera par donner des agréments à des bourrécoles } \\
\text { où on apprendra à faire avancer et arrêter l'animal. (...)Mais comment yal khaoua } \\
\text { fera-t-il l'âne du Centre pour comprendre les ordres de son maître de l'Est, si celui } \\
\text { de l'Ouest ne parle pas la même langue que son frère du Sud, et vice-versa? C'est } \\
\text { très simple. Il faut opter pour une seule langue dans l'ânation. Tous les ânes se } \\
\text { verront recycler (...). Ils iront dans les écoles, ils passeront par l'apprentissage et la } \\
\text { mise à niveau afin d'obtenir le permis de " bourricoler " (28/05/2009). }\end{array}$ \\
\hline
\end{tabular}




\begin{tabular}{|c|c|c|}
\hline $\begin{array}{l}\mathrm{N}^{\circ} 2 . \\
\text {-asse } \\
\text {-ation } \\
\text {-eur }\end{array}$ & $\begin{array}{l}\text { Creusasse } \\
\text { Creuseur } \\
\text { piétonnation }\end{array}$ & $\begin{array}{l}\text { "Creusez, fouillez, bêchez; ne laissez nulle place où la main ne passe et repasse, } \\
\text { écrivait La Fontaine. Depuis, sans trop se creuser les méninges, on creuse. (...) } \\
\text { Oran, ville de la creuse. Il n'y a pas un quartier, une artère qui échappe à la } \\
\text { "creusasse". Des crevasses. Partout. (...) Notre ville ressemble à une baghrira avec } \\
\text { beaucoup de miel pour les creuseurs en chef (...).Alors creusons. Ma ville est le } \\
\text { creuset des creuseurs. El haffara. (...)Les chauffeurs n'ont qu'à faire attention. Il } \\
\text { leur est demandé plus de vigilance. Faire attention aux piétons qui traversent } \\
\text { défiant toutes les règles de la "piétonnation". (...)Amala "creusez, fouillez, bêchez; } \\
\text { (...)». Un trésor est caché... dans le budget des villes. Creusez! Défoncez!» } \\
(17 / 02 / 2009) \text {. }\end{array}$ \\
\hline $\begin{array}{l}\mathrm{N}^{0} 5 . \\
\text {-eur }\end{array}$ & parkingueur & $\begin{array}{l}\text { "Tout baigne dans l'huile», selon la bonne et vieille réflexion populaire, oui mais } \\
\text { pour qui? Pas en tous cas pour le pauvre consommateur saigné à blanc, comme } \\
\text { un veau (rien) par la Sonelgaz, l'ADE, les impôts, el kra de l'OPGI, les PTT } \\
\text { (téléphone), le boucher, le poissonnier, le vendeur de lait et même par le } \\
\text { "parkingueur! Mtargui patron du trottoir»!»(15/10/2009). }\end{array}$ \\
\hline $\begin{array}{l}\mathrm{N}^{\circ} 7 . \\
\text {-erie } 85\end{array}$ & & $\begin{array}{l}\text { "La jarerie: (...) Qu'il vente ou qu'il neige, elle est en poste, très tard sur son } \\
\text { balcon, pour contrôler les derniers va-et-vient fel houma, pour se réveiller très tôt } \\
\text { le lendemain se poster sur le palier de l'immeuble au rez-de-chaussée.(...) C'est le } \\
\text { nouveau voisin qui descend. (...) «Bonjour jari, mzia le bon Dieu vous a envoyé à } \\
\text { nous. On avait peur que l'appartement soit occupé par des jirène pas très bien. (...) } \\
\text { Dieu ouassa aala le jar et un jar de votre trempe... » (27/08/2009). }\end{array}$ \\
\hline
\end{tabular}

Exemples $\mathrm{N}^{\circ} 1$ : la formation des néologismes est affichée dans le sens ou sa dérivation est explicite :

- « $\left(l^{\prime}\right)$ ânation » : radical : « $\left(l^{\prime}\right)$ âne $(s)$ » répété trois fois avant d'engendrer le dérivé « (l') ânation », par ajout du suffixe -ation, pour signifier « action par laquelle se forment l'âne - les ânes, ou le résultat de cette action qui vise l'âne, moyen de locomotion, et qui prend place dans un champ lexical constitué de "l'âne», «les ânes», "dos d'âne», "l'animal». Notons qu'inséré dans son contexte phrastique, «il faut opter pour une seule langue dans l'ânation », le lecteur entend, encouragé entre autre par l'homophonie, «la nation» dont le choix d'une seule langue a suscité, en Algérie, un grand débat répercuté par les médias. Ainsi le sens de la lexie se complique : d'une part, former / « recycler » les ânes dans une seule langue est une consigne ( il faut») dictée par la nécessité de communiquer du Centre au Sud, de l'Est à l'Ouest; d'autre part, l'appliquer c'est entreprendre un processus d'«ânation» de la nation dans le sens où «ânation» (et la répétition d' «âne(s)»), fait entendre par association phonico-sémantique : « ânonner ».

Ainsi, dans le langage idiosyncrasique du chroniqueur, «l'ânation» se lit aussi comme l'action (ou le résultat) d' " ânonner ». L'action de "l'ânation » concerne donc "les ânes » qui devront aller dans les écoles pour apprendre à «bourricoler», autre néologisme de la chronique.

- «Bourricoler » : le radical semble aisé à identifier : «bourricot ». Le suffixe, formé du suffixe er, est en principe ajouté au radical pour former le verbe. Dans ce cas, la lexie formée aurait été « bourricoter » (?!).

Or, pour former «bourricoler », il nous faut considérer le mot «école » qui apparaît dans l'environnement phrastique de «bourricoler» et qui entretient avec lui une relation lexico-sémantique : il s'agit du lieu où se fera cet apprentissage : «Ils iront dans 
les écoles, ils passeront par l'apprentissage et la mise à niveau afin d'obtenir le permis de "bourricoler" ".

Pour les (mauvais) conducteurs, le chroniqueur a prévu des «bourrécoles». Ce néologisme est formé par composition (voir ce procédé infra) : il est constitué de la coalescence de deux mots de registres différents: «bourré" (argot français pour signifier «ivre » au sens figuré) et «école ». L'identité de la voyelle /é/ à la finale du premier mot et à l'initiale du second a favorisé leur fusion par contraction.

Le sens de "bourré" est induit du texte par association: «(...) obtenir le permis de "bourricoler" » fait écho, en amont, dans la même chronique, à « (...) retrait de permis pour conduite en état de buveresse", suivi de "ainsi, on commencera par donner des agréments à des bourrécoles où on apprendra à faire avancer et arrêter l'animal. Ces écoles, ces bourrécoles initieront les candidats à la conduite».

De plus «bourré" apparaît au début de la chronique : "Toutes nos villes sont bourrées de cités qui n'ont rien à envier aux kouris d'antan ".

Le sens de «bourré » est renforcé par la proximité syntagmatique de « (retrait de permis pour conduite en état de "buveresse" ». Cette expression, lexicalisée, permet de superposer "buveresse » et "ivresse " en laissant entendre "buveur ", associé à "beuverie ». Le néologisme combine ainsi deux procédés, composition et troncation de "buveur » et d' "ivresse ", pour former un mot qui signifie, dans le langage propre du chroniqueur, «l'état de celui qui aime boire, en groupe, des boissons alcoolisées jusqu'à l'ivresse » / "état de buveresse» / " bourrés ».

Les "bourrécoles" sont des " écoles " pour "bourrés ", "chauffards", etc., et les " bourricoles ", des écoles pour « bourricots».

Mais le «bourré »/《chauffard»/ « qui ne sait pas conduire » et le «bourricot» sont tous deux des «candidats à la conduite» / « apprentissage...pour obtenir le permis». Leur similitude tend vers leur identité puisque celui qui «ne sait pas ... conduire», «le chauffard», est, au sens figuré, un «bourricot " ('inverse n'est pas du tout envisagé). Mais, cette assimilation, fortement suggérée par le chroniqueur, ne va pas au-delà de la similitude; le chroniqueur ayant insisté sur les fonctions de cet «animal» dans la société ; d'où la séparation des lieux d'apprentissages, malgré leur ressemblance à la fois sémantique et phonique : une " bourrécole » n'est pas tout à fait une " bourricole ».

Même si se sont toutes deux des "écoles", le chroniqueur ménage une nuance sémantique entre les deux néologismes.

Dans les exemples de néologismes du $\mathrm{N}^{\circ} 1$ que nous avons passés en revue, nous constatons donc que la dérivation se construit de façon explicite, sous les yeux, en quelque sorte, du lecteur.

Dans l'exemple $\mathrm{N}^{\circ} 2$ (voir annexe), le néologisme "piétonnation» est dérivé du substantif «piétons » qui le précède auquel est ajouter le suffixe « ation » pour signifier l'action anarchique des " piétons » «qui traversent défiant toutes les règles de la ... »; d'où l'association phonético-sémantique de " piétonnation » avec " piétiner » dans le sens de "fouler aux pieds» («les règles»). "Piétonnation» ne se donne donc pas à lire simplement comme l'acte de marcher des " piétons ", mais comme l'acte de marcher en foulant aux pieds les règles, en les " piétinant».

C'est d'ailleurs dans ce sens et autour du même thème (la conduite) qu'est formé, en aval (voir exemple $\mathrm{N}^{\circ} 3$ en annexe), près de deux ans après, le néologisme "piétineurs", s'agissant cette fois des (mauvais) automobilistes: "Cet objet qui parait anodin, porté durant toute la durée du voyage, rappellera à l'ordre les kamikazes grilleurs de priorités, doubleurs n'importe comment, « piétineurs » de lignes jaunes et j'en passe ». 
«Piétineurs » actualise ici, pour les automobilistes, son sens de " défi aux règles » attaché aux «piétons", et souligné par les deux autres néologismes contigus, "grilleurs » et "doubleurs"; les trois qualifiant respectivement un aspect des "kamikazes» de la route. Ce thème est très souvent traité par le chroniqueur et toujours dans le même sens. Dans l'exemple $\mathrm{N}^{\circ} 4$ (voir annexe), la caractérisation des (mauvais) automobilistes lui permet de créer un nouveau néologisme en -isme à côté de celui en -eur : "il y a le tapageur "choufni » (...). Kayene l'autre qui fait dans le khéchinisme, qui vient se placer sur la voie de droite pour vous doubler et vous faire une queue de poisson lorsque le feu tournera au vert ». Dans la chronique, « khéchinisme » prend place dans le champ lexical de " cinglés - mhabile - tapageur/choufni» etc. Ces mots entretiennent des relations lexicosémantiques explicites : correspondance dans les deux langues pour les uns (tapageur/ choufni), équivalence phonético-prosodique affichée pour d'autres : «automobile rime avec débile et " $m$ 'habile" ".

Or, les « cinglés » sont des gens tout à fait ghaya dans leur peau, bien de leur personne, [qui] se transforment en cinglés dès qu'ils ont (...) une machine entre les mains ». Ainsi est ménagée dans la chronique, la correspondance entre " cinglés » et "m'habile » qui " rime » avec " automobile » et " débile ». De plus les " cinglés » est le terme générique qui désigne autant le «tapageur » qui abuse du « clignotant » que « l'autre qui fait dans le khéchinisme ", spécialisé dans les "queues de poissons ». Ce rapprochement lexicosémantique entre «cinglés» / "automobile» / «débile» / «m'habile» / "tapageur" / "khéchi-nisme» induit une lecture spécifique du néologisme "khéchinisme». Le suffixe "-isme" est ajouté après le radical de l'arabe dialectal «khéchin ».

Le chroniqueur a pris le soin, comme on l'a souligné à propos d'autres néologismes, de ménager sa compréhension en français puisque le comportement visé est de "cinglé». Mais le distinguer du "tapageur» qui compte aussi parmi les «cinglés», sa contiguïté phonético-sémantique avec "automobile», "débile», "m'habile» va imposer son association avec "crétinisme»; déficience mentale déjà convoquée par "débile». Le radical en arabe "khéchin " / rustre renforce les sèmes de /brutalité/ grossièreté/. Le " khéchinisme ", dans le contexte de Tranche de vie, est le comportement d'un automobiliste « cinglé», "m'habile », « débile », « crétin », et rustre.

Nous soulignerons ici, la cohérence sémantique des néologismes créés par le chroniqueur par-delà le temps qui sépare leur apparition.

Toujours dans l'exemple $\mathrm{N}^{\circ}$ 2, le néologisme "creusasse » est généré, en texte, par le verbe «creuser » apparu plusieurs fois en amont dans la chronique : «creusez» - "se creuser » - " on creuse » - «la creuse ».

Dans le passage du radical «creuser » au néologisme, le chroniqueur a ménagé un relai par sa substantivation, "la creuse » pour désigner l'acte de creuser. Ce substantif est suivi de "creusasse " qui apparaît en étroite contigüité avec "crevasse " homophone. Cette distribution syntagmatique de lexies en relations lexico-sémantiques, induit la signification de "creusasse », formé du radical «creuse » et du suffixe dépréciatif -asse; le sens du radical s'imposant dans l'excès de sa répétitions que le suffixe empreint de sa péjoration. Le nom "creusasse " signifie en contexte le résultat de l'action de creuser avec excès ("la creuse ») "des crevasses" "partout» dans "la ville d'Oran"; d'où son surnom : « la ville de la creuse » synonyme de " la ville de la creusasse » et de «creuset des creuseurs». El haffara». "Creuser» a donc généré dans le même syntagme son homonyme, "creuset » en l'intégrant à son champ lexical, et son nom d'agent dans les deux langues, «creuseurs " - «el haffara ». Ce nom d'agent, "creuseurs », est associé à 
son apparition immédiatement en amont, dans « Notre ville ressemble à une baghrira avec beaucoup de miel pour les creuseurs en chef", pour faire de "la creusasse " une opération (illicite) rentable pour ses «creuseurs en chef " mais pas pour les «chauffeurs » ni «les piétions ». Ainsi, « la creusasse », dans le « jargon » du chroniqueur, consiste à «creuser » «des crevasses », « partout » dans les « quartiers » et les « artères » de « la ville», pour tirer profit du «trésor caché »/ « budget de la ville», au détriment des usagers.

8 La suffixation, est, en général, dans "Tranche de vie», un processus de création néologique par auto-engendrement ou auto-génération dans la chronique même. $\mathrm{Ce}$ processus peut aller du radical vers le néologisme, comme c'est le cas ci-dessus, ou du néologisme vers le radical, comme dans l'exemple $\mathrm{N}^{\circ} 7$ (voir annexe) avec le suffixe erie.

Le néologisme «jarerie » accompagné de l'article « la», « la jarerie », constitue le titre de la chronique dans laquelle son sens est expliqué à deux niveaux. Le premier impose le radical d'un point de vue formel : «jar », repris plusieurs fois dans la chronique, même dans sa traduction en français, et dans l'ordre suivant : « (le nouveau) voisin » - " (au nouveau) voisin » - «jari »- «jirène » - «le jar » - « un jar » - (un) voisin ». Contrairement à " voisin ", qui apparaît au singulier, le radical en arabe, « jar ", se décline au singulier, au pluriel, avec l'article défini, indéfini, le possessif. Le second concerne l'action visée par le néologisme lui-même, "jarerie ", qui est pourtant absent de la chronique, à travers son agent nommée à deux reprises, "El gachoura», et dont l'action est amplement décrite. Celle-ci consiste à la fois à espionner ses voisins, à les harceler, à jaser à leur sujet tout en prêtant, hypocritement, ses réflexions à d'autres de ses voisins. C'est ainsi, que favorisée formellement et sémantiquement par la proximité de « jar » et de « voisin », la superposition de « jarerie » à « jaserie » est mise au jour, pour signifier que la "jarerie» est la qualité d'un voisinage caractérisé par la "jaserie» hypocrite. Ce procédé de création néologique par auto-engendrement des signifiants n'est pas exclusif.

Lorsque le néologisme, constitué d'un nom d'agent, n'affiche pas sa formation, il peut être suivi de son explication/définition, comme dans le cas suivant :

\begin{tabular}{|l|l|l|}
\hline $\begin{array}{l}\mathrm{N}^{\circ} \text { 5. } \\
\text {-eur }\end{array}$ & parkingueur & $\begin{array}{l}\text { "Tout baigne dans l'huile», selon la bonne et vieille réflexion populaire, oui mais pour } \\
\text { qui? Pas en tous cas pour le pauvre consommateur saigné à blanc, comme un veau } \\
\text { (rien) par la Sonelgaz, l'ADE, les impôts, el kra de l'OPGI, les PTT (téléphone), le boucher, } \\
\text { le poissonnier, le vendeur de lait et même par le «parkingueur! Mtargui patron du } \\
\text { trottoir»!» (15/10/2009). }\end{array}$ \\
\hline
\end{tabular}

Pour "parkingueur", le radical parking est un anglicisme courant aussi bien en français ${ }^{8}$ qu'en arabe dialectal ou en berbère. A ce radical, le chroniqueur ajoute le suffixe d'origine latine -eur pour former le nom d'agent, "parkingueur ", qu'il fait suivre non seulement de sa traduction en arabe dialectal mais aussi de sa définition spécifique : « «parkingueur! Mtargui patron du trottoir ». La profession de " parkingueur » devrait être liée au radical «parking».

Or, la définition en français substitue " parking » à «trottoir » et "gardien », convoqué par association devient, dans la chronique, "patron» qui induit, toujours par association, la superposition de "trottoir» à "entreprise». "Parkingueur » se donnerait à lire dans son sens de " patron d'entreprise ». 
Le nom d'agent «parkingueur »-« patron de trottoir »/ «entreprise» est accrédité, dans le sens que lui confère la chronique, en clôturant le champ lexical pléthorique de tous les noms d'agent cités dans la chronique et qui ont en commun de «saigner à blanc le pauvre consommateur » : «la Sonelgaz, l'ADE, les impôts, el kra de l'OPGI, les PTT (téléphone), le boucher, le poissonnier, le vendeur de lait et même le ... ».

Parmi eux, "parkingueur » est l'objet de renchérissement puisque classé dernier. Cette place est d'autant plus dépréciée que le chroniqueur reprend cette liste, immédiatement en aval, pour compléter l'énumération des agents dont le consommateur est victime: "Vous remarquerez qu'on n'évoque pas ici le tailleur (...), le boulanger (...), le mécanicien (...), le peintre (...),le cordonnier (...), le spécialiste de l'électroménager (...),on n'arrivera pas jusqu'au bijoutier (...). On n'oubliera pas non plus le marchand de légumes (...). Mais, à la faveur de ce contexte, truffé de noms d'agent, et de cette gradation (" et même ...»), le "parkingueur » est imposé en tant que nom d'agent dérivé de " parking »; la consonne vélaire /g/ étant préservée en français par l'ajout du /y/ («u»).

Son synonyme, "mtargui », contigu, renforce le sème de /pouvoir/ attaché à ce nom d'agent, dans le sens d'autoritarisme, amplifiant ainsi jusqu'à la dérision le paradoxe entre la fonction de « patron » et le dérisoire de l'entreprise (« trottoir »).

Les cas de suffixation étant très nombreux, il nous est impossible de les passer en revue de façon exhaustive. Par ailleurs, là n'était pas notre objectif. Nous voulions montrer que quel que soit le type de suffixation, le néologisme formé acquiert toujours, en plus du sens inhérent à son radical, des sèmes spécifiques en contexte qui justifient la création néologique et l'accrédite. L'observation est également valable pour la préfixation, comme montré supra, et la formation parasynthétique abordée dans le point suivant.

La formation parasynthétique :

Ce processus de formation des mots, qui consiste à ajouter simultanément, à un mot, un préfixe et un suffixe nominal ou verbal, est peu productif dans les chroniques journalistiques de notre corpus. Seules quelques lexies néologiques sont obtenues sur ce modèle, comme démoustification. Sur le plan typographique, il est intéressant de signaler que le chroniqueur n'a pas utilisé les marques particulières pour distinguer ses lexies néologiques. Comme nous pouvons le constater dans l'exemple ci-dessous, la lexie inventée démoustification n'a pas été mise entre guillemets alors qu'elle est associée à la lexie "démoustication" attestée dans le dictionnaire Le Petit Robert électronique 2012: "Ils sont là, ils sont partout et c'est pourquoi il faut s'y méfier des moustiques car même l'hiver est leur saison. Pourtant, il suffirait d'une petite campagne de démoustification pour rendre la vie plus agréable au citoyen [...]» (20/07/2009). Mais la lexie est aussi associée à "mystification » sur les deux plans, formel et sémantique. Donc, " une campagne de démoustification " vise la lutte à la fois contre les moustiques et la mystification pour un même objectif : «rendre la vie plus agréable au citoyen ».

9 Cette analyse partielle de la néologie journalistique, nous a tout de même permis d'entrevoir les mécanismes d'élaboration de nouvelles lexies par affixation. Le plus important est celui de la génération du néologisme par auto-engendrement explicite du radical vers la lexie ou l'inverse, fondé sur le tissage, dans le texte de la chronique, ou dans ceux de plusieurs chroniques reliées entre elles, de champs lexico-sémantiques qui autorisent la construction et la production de sens des lexies. Celui-ci est la combinaison du sens avéré du radical et de celui spécifique acquis en contexte; d'où le 
phénomène d'innovation lexicale qui caractérise le langage idiosyncrasique de l'auteur qui tout en utilisant des néologismes qui existent déjà, en crée de nouveaux.

Ce bref aperçu analytique nous a également permis d'illustrer des cas de coalescence d'éléments de langues différentes dans la formation de nouvelles lexies. Ainsi, la chronique journalistique d'El Guellil, comme nous avons tenté de le montrer, en combinant généralement les deux langues, l'arabe et le français, s'adapte parfaitement à la réalité sociolinguistique et socioculturelle de la société algérienne contemporaine. Pourtant la suffixation est, d'après nos statistiques et comme signalé plus haut, peu répandue. S'agissant de la préfixation et de la suffixation, nous avons remarqué que la dérivation était essentiellement savante (d'origine latine) d'où des opérations d'interprétation sémantique souvent fastidieuses (action, résultat de l'action, etc.) incompatibles avec le genre journalistique de la chronique. Si le chroniqueur s'y incarne, il ne s'y enferme pas : son objectif reste la séduction de son lecteur. Selon Gilbert Lavoie cité par J.C. Picard (op. cit. : 42), il doit « savoir prendre les lecteurs par la main, être capable de leur faire vivre l'événement, de leur montrer l'être humain derrière toutes les histoires, de faire ressortir ses angoisses, ses difficultés, ses joies et ses peines. C'est la qualité la plus fondamentale». Dans ce qui est envisagé comme une entreprise d'accompagnement, voire d'assistance du lecteur, le chroniqueur se présente comme un facilitateur de lecture des réalités sociales; d'où l'incompatibilité de l'affixation telle qu'évoquée avec le genre de la chronique. Ce n'est pas le cas pour certains autres procédés de la néologie (voir tableau des procédés avec pourcentages correspondant en annexe 2).

\section{BIBLIOGRAPHIE}

BENMAYOUF Ch.-Y, Renouvellement social, renouvellement langagier dans l'Algérie d'aujourd'hui, L'Harmattan, 2008.

BASTUJI J, (1979), « Notes sur la créativité lexicale », in Adda (R.) et ali, Néologie et Lexicologie. Paris. Larousse, 1979, pp. 12-20.

BENZAKOUR F, « Le Français dans la réalité marocaine. Faits d'appropriation. L'exemple de l'écart lexical ", in Par monts et par vaux : itinéraires linguistiques et grammaticaux : mélanges de linguistique générale et française, Editions, Peeters. Louvain-Paris, 2001, 455 pages.

CATARIG A.-T, 2011, « Néologismes d'auteur » dans la presse écrite généraliste, in Professional communication and translation studies, (1-2). Disponible sur le site :

< http://www.cls.upt.ro/files/conferinte/proceedings/PCTS > < https://sc.upt.ro/images/ cwattachments/116_0644bee096587bca1332ff4daf86dff4.pdf $>$

CHARAUDEAU P, Grammaire du sens et de l'expression, Paris, Hachette Education, 1992.

DUBOIS J. et al., Dictionnaire de linguistique, Larousse, 1973. HUMBLEY J, « Evolution du lexique », Histoire de la langue française 1945-2000, Paris : CNRS, 2000, PP71-106. 
PICARD J.-C, « La chronique dans les quotidiens québécois : un genre journalistique de plus en plus populaire ", in Les Cahiers du journalisme, n6, 1999, pp. 36-49. Disponible sur le site : < http:// www.cahiersdujournalisme.net/pdf/06/06_Picard.pdf >

SABLAYROLLES J.-F, 2012, « Des néologismes par détournement? ou Plaidoyer pour la reconnaissance du détournement parmi les matrices lexicogéniques », in Actes du colloque DORIF Disponible sur le site :

< http://hal.archivesouvertes.fr/index.php?

halsid=fbj4oni80e5676qce7n1rcmpb6\&view_this_doc=halshs-00735933\&version=1 $><$ https:// hal.archives-ouvertes.fr/halshs-00735933 >

SABLAYROLLES J.-F, La néologie en français contemporains. Examens du concept et analyse de productions néologiques récentes, Paris, Honoré Champion, 2000.

SABLAYROLLES J.-F, 2002, « Fondements Théoriques des Difficultés Pratiques Du Traitement Des Néologismes ", in Revue française de linguistique appliquée. Vol VII-1. « Lexique : recherche actuelles », Disponible sur le site :

$<$ https://www.cairn.info/revue-francaise-de-linguistique-appliquee-2002-1-page-97.html >

SABLAYROLLES J.-F, « Néologismes : une typologie des typologies ». Disponible sur le site :

$<$ http://www.eila.univ-paris-diderot.fr/_media/recherche/clillac/ciel/cahiers/

96-97/1sablayrollestexte.pdf >

SADER FEGHALI L, «La presse vue à travers néoscope : quand les contextes médiatiques sont mis au service de la néologie ", in Mots, Termes et Contexte, Actes des septièmes journées scientifiques du réseau de chercheurs, Lexicologie Terminologie Traduction, Bruxelles, Belgique, 2005, pp. 525-534.

\section{ANNEXES}

Annexe 1 : cas de suffixation

Suffixes Néologismes Illustration




\begin{tabular}{|c|c|c|}
\hline $\begin{array}{l}\mathrm{N}^{\mathrm{O}} 2 . \\
\text {-asse } \\
\text {-eur } \\
\text {-ation } \\
\text {-isme }\end{array}$ & $\begin{array}{l}\text { Creusasse } \\
\text { Creuseur } \\
\text { piétonnation } \\
\text { piétineur } \\
\text { parkingueur }\end{array}$ & $\begin{array}{l}\text { "Creusez, fouillez, bêchez; ne laissez nulle place où la main ne passe } \\
\text { et repasse, écrivait La Fontaine. Depuis, sans trop se creuser les } \\
\text { méninges, on creuse. (...) Oran, ville de la creuse. Il n'y a pas un } \\
\text { quartier, une artère qui échappe à la "creusasse". Des crevasses. } \\
\text { Partout. (...) Notre ville ressemble à une baghrira avec beaucoup de } \\
\text { miel pour les creuseurs en chef (...).Alors creusons. Ma ville est le } \\
\text { creuset des creuseurs. El haffara. (...)Les chauffeurs n'ont qu'à faire } \\
\text { attention. Il leur est demandé plus de vigilance. Faire attention aux } \\
\text { piétons qui traversent défiant toutes les règles de la "piétonnation". } \\
\text { (...)Amala "creusez, fouillez, bêchez; (...)». Un trésor est caché... dans } \\
\text { le budget des villes. Creusez! Défoncez!» (17/02/2009). } \\
\text { "Tout baigne dans l'huile », selon la bonne et vieille réflexion } \\
\text { populaire, oui mais pour qui ? Pas en tous cas pour le pauvre } \\
\text { consommateur saigné à blanc, comme un veau (rien) par la Sonelgaz, } \\
\text { l'ADE, les impôts, el kra de l'OPGI, les PTT (téléphone), le boucher, le } \\
\text { poissonnier, le vendeur de lait et même par le «parkingueur! } \\
\text { Mtargui patron du trottoir »!» (15/10/2009). }\end{array}$ \\
\hline $\begin{array}{l}\mathrm{N}^{\mathrm{O}} 3 . \\
\text {-eur }\end{array}$ & $\begin{array}{l}\text { grilleurs } \\
\text { doubleurs } \\
\text { piétineurs }\end{array}$ & $\begin{array}{l}\text { "Commençons déjà par le commencement. Obliger le chauffeur à } \\
\text { porter la ceinture de sécurité, c'est lui rappeler, déjà, qu'il transporte } \\
\text { des êtres humains. Qu'un accident est vite arrivé. Cet objet qui paraît } \\
\text { anodin, porté durant toute la durée du voyage, rappellera à l'ordre } \\
\text { les kamikazes grilleurs de priorités, doubleurs n'importe comment, " } \\
\text { piétineurs » de lignes jaunes et j'en passe. Ceux qui sillonnent nos } \\
\text { routes. Nos routes mortelles" (31/03/2011). }\end{array}$ \\
\hline $\begin{array}{l}\mathrm{N}^{\mathrm{O}} 4 . \\
\text {-eur } \\
\text {-isme }\end{array}$ & $\begin{array}{l}\text { tapageur } \\
\text { khéchinisme }\end{array}$ & $\begin{array}{l}\text { «Ils ont beau écrire, ils ont beau dénoncer. Les drames se suivent et } \\
\text { se ressemblent. (...) Fi youm mina el ayam, il faudra qu'on m'éclaire. } \\
\text { En vertu de quoi, de quel principe, de quelle vertu, nass, des gens tout } \\
\text { à fait ghaya dans leur peau, bien de leur personne, se transforment } \\
\text { en cinglés dès qu'ils ont un appareil, ou une machine entre les } \\
\text { mains? Pourquoi automobile rime avec débile et " m'habile »? Ils } \\
\text { sont pressés d'arriver nulle part, conduisant hargne au ceeur, comme } \\
\text { si tout le monde était contre eux. Il y a le tapageur " choufni » qui } \\
\text { attend le dernier moment pour freiner sec à un feu rouge et qui } \\
\text { démarre en cow-boy (à chacun ses origines) dès que le vert yaghmez. } \\
\text { Kayene l'autre qui fait dans le khéchinisme, qui vient se placer sur la } \\
\text { voie de droite pour vous doubler et vous faire une queue de poisson } \\
\text { lorsque le feu tournera au vert. Lakhor qui, dans un embouteillage, } \\
\text { change cent fois de voie dans l'espoir d'aller plus vite. (...). }\end{array}$ \\
\hline $\begin{array}{l}\mathrm{N}^{0} 7 . \\
\text {-erie } 85\end{array}$ & la jarerie & $\begin{array}{l}\text { "La jarerie: (...) Qu'il vente ou qu'il neige, elle est en poste, très tard } \\
\text { sur son balcon, pour contrôler les derniers va-et-vient fel houma, } \\
\text { pour se réveiller très tôt le lendemain se poster sur le palier de } \\
\text { l'immeuble au rez-de-chaussée.(...) C'est le nouveau voisin qui } \\
\text { descend. (...) « Bonjour jari, mzia le bon Dieu vous a envoyé à nous. } \\
\text { On avait peur que l'appartement soit occupé par des jirène pas très } \\
\text { bien. (...) Dieu ouassa aala le jar et un jar de votre trempe... " } \\
(27 / 08 / 2009) .\end{array}$ \\
\hline
\end{tabular}


Annexe 2 : Tableau des procédés de création néologique dans « tranche de vie » de 2009 à 2014

\begin{tabular}{|c|c|c|}
\hline \multicolumn{3}{|c|}{ 1. Matrices néologiques internes $(82,03 \%)$} \\
\hline \multirow{4}{*}{$\begin{array}{l}1.1 \text { La matrice } \\
\text { morphosémantique : } \\
52,86 \% \text {. }\end{array}$} & \multicolumn{2}{|c|}{$\begin{array}{l}\text { 1.1.1 Affixation ou dérivation morphologique }(08,11 \% \text {.) : } \\
\text { préfixation, suffixation, formation parasyntaxique (cf. article } \\
\text { supra) }\end{array}$} \\
\hline & $\begin{array}{l}\text { 1.1.2. Composition } \\
\text { (a) : } 31,24 \% \\
\text { - synapsie(b) (12, } \\
03 \%)\end{array}$ & $\begin{array}{l}\text { a- « la grippe financière et la crise porcine " } \\
(12 / 08 / 2009) \text { - « diplômé- chômeur » } \\
(05 / 02 / 2011) \text { - «photolangage » } \\
(23 / 03 / 2009)-\text { «pneumanie » } \\
(29 / 09 / 2009)- \\
\text { b- «Panne à fric » }(15 / 06 / 2009) \text { - « bête de } \\
\text { route »(20/05/2009) - } \\
\text { "pauvres de Bill Gattes » }(09 / 08 / 2011)-\end{array}$ \\
\hline & $\begin{array}{l}\text { 1.1.3. Mots-valises } \\
(13,14 \%)\end{array}$ & $\begin{array}{l}\text { " boujouterie » }(30 / 06 / 2014)-\text { " } \\
\text { pharmafruit » }(20 / 06 / 2014)- \\
\text { « dictarchie » }(15 / 11 / 2009)\end{array}$ \\
\hline & $\begin{array}{l}\text { 1.1.4. Composés } \\
\text { hybrides : } 22 \text {, } \\
49 \% \text {. }\end{array}$ & $\begin{array}{l}\text { «Opium halal « }(07 / 02 / 2010) \text { - « Harraga } \\
\text { numériques » }(19 / 02 / 2012)- \\
\text { « Mendicité new-look»(03/10/2009) - }\end{array}$ \\
\hline & $\begin{array}{l}\text { 1.1.5. Imitation et } \\
\text { déformation } \\
13.40 \%\end{array}$ & $\begin{array}{l}\text { - « rav-âgé, El-Guellil chref» }(22 / 06 / 2014)- \\
\text { «deuv, zdreuv, zdreuv- tac» } »(04 / 07 / 2008 / \\
-\end{array}$ \\
\hline \multirow{4}{*}{$\begin{array}{l}1.2 \text {.La matrice } \\
\text { syntaxico- } \\
\text { sémantique : } 15,78 \% \text {. }\end{array}$} & $\begin{array}{l}1.2 .1 . \\
\text { Adjectivation (a) }\end{array}$ & \multirow{4}{*}{$\begin{array}{l}\text { a - «basketté » }(25 / 03 / 2011)-b-0 \\
\mathrm{c}-\text { « Du raï à la déraille » }(04 / 10 / 2010)- \\
\mathrm{d}-\text { « se touristent }(29 / 08 / 2009)- \\
\text { «catharsiser }(16 / 02 / 2009)-\end{array}$} \\
\hline & $\begin{array}{l}1.2 .2 \text {. } \\
\text { Adverbialisation } \\
\text { (b) }\end{array}$ & \\
\hline & $\begin{array}{l}\text { 1.2.3. } \\
\text { Nominalisation (c) }\end{array}$ & \\
\hline & $\begin{array}{l}1.2 .4 \text {. } \\
\text { Verbalisation }(d)\end{array}$ & \\
\hline \multirow{4}{*}{$\begin{array}{l}\text { 1.3. La matrice } \\
\text { morphologique : } \\
9,83 \% \text {. }\end{array}$} & 1.3.1. Siglaison $(a)$ & \multirow{4}{*}{ 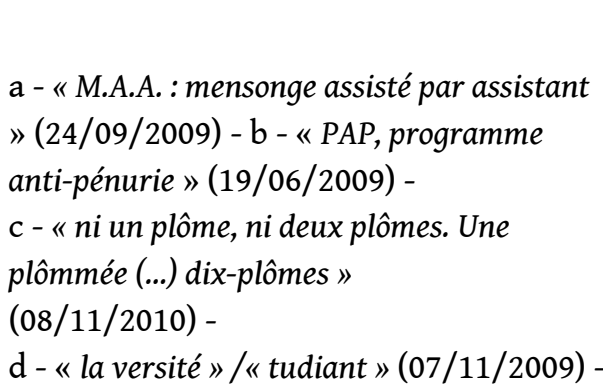 } \\
\hline & $\begin{array}{l}\text { 1.3.2. Acronymie } \\
\text { (b) }\end{array}$ & \\
\hline & $\begin{array}{l}\text { 1.3.3. Troncation } \\
\text { par apocope (c) }\end{array}$ & \\
\hline & & \\
\hline
\end{tabular}




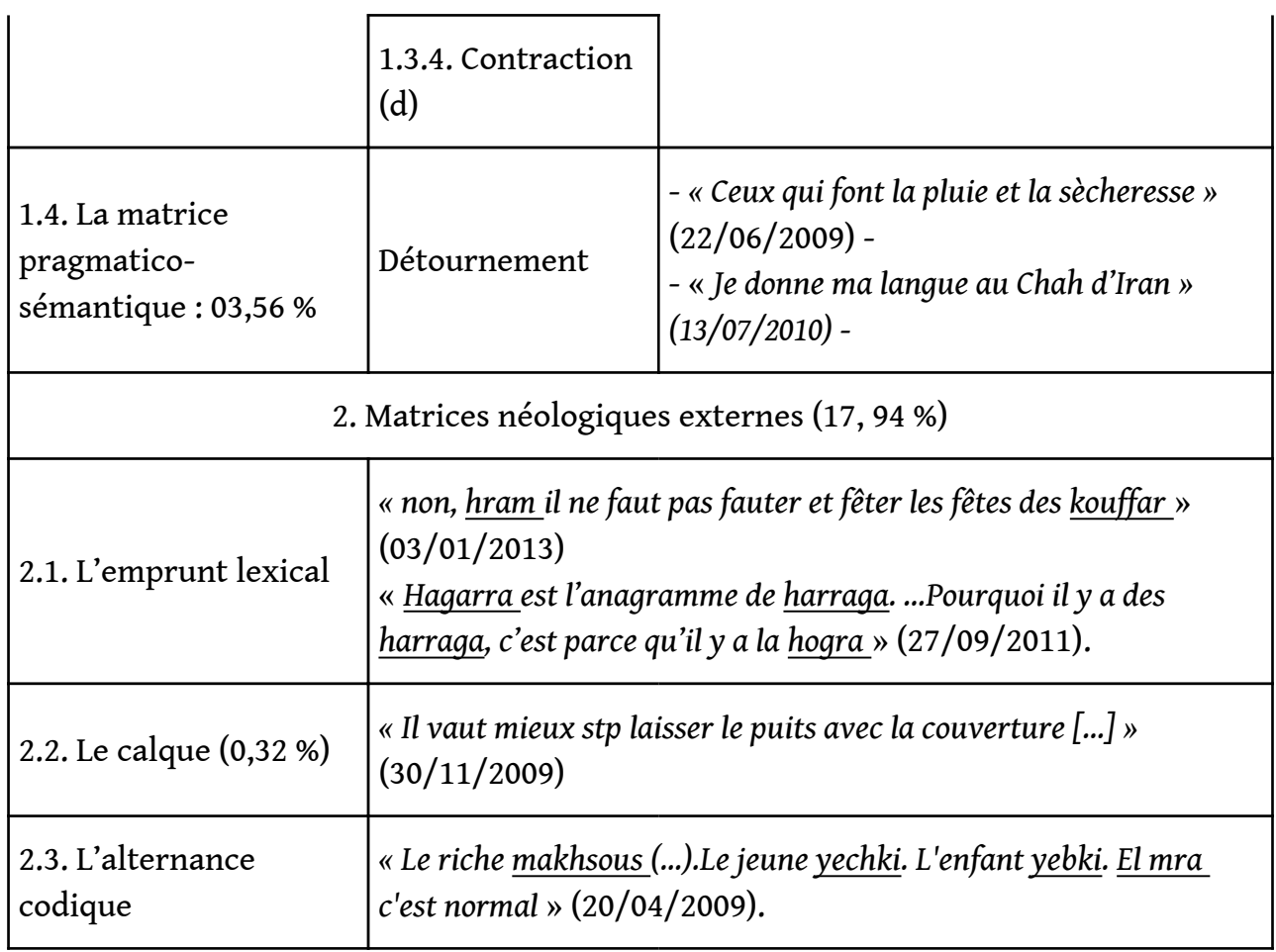

\section{NOTES}

1. «El-Guellil » est une transcription en caractères latins du mot de l'arabe dialectal qui signifie «le pauvre».

2. Les Cahiers $d u$ journalisme, $\mathrm{N}^{\circ} 6$, octobre 1999, p.38. Consulté sur le site: http:// www.cahiersdujournalisme.net/pdf/06/06_Picard.pdf

3. Pour ce genre d'étude, on ne peut consulter que les quelques ouvrages d'usage courant, remis régulièrement à jour, largement répandus et utilisés dans la vie courante.

4. Cf. par exemple La Néologie journalistique, mémoire de Magister d'Adaci Sana soutenu en 2008 sous la direction de J.-F. Sablayrolles. Consultable sur le site : < https://bu.umc.edu.dz/theses/ francais/ADA1011.pdf

5. J-F Sabayrolles propose d'établir sa propre typologie des procédés de formation, en apportant de légères modifications aux procédés de formations appelés, depuis J. Fournier, "matrices lexicogéniques ». Sa typologie, organisée en deux grandes matrices, internes et externes, est présentée dans un article intitulé "Néologismes: une typologie des typologies ", in Cahiers du CIEL 1996-1997, Centre d'études en lexicologie - UFR E.I.L.A. - Université Paris 7 Denis Diderot, pp.11-48. Consultable sur le site: http://www.eila.univ-paris-diderot.fr/_media/recherche/ clillac/ciel/cahiers/96-97/1sablayrollestexte.pdf

6. «Dans la terminologie de B. Pottier, est l'unité de comportement lexical. Elle est opposée au morphème, plus petit signe linguistique, et au mot, unité minimale construite. C'est donc l'unité fonctionnelle significative du discours. La lexie simple peut être un mot (...). La lexie composée peut contenir plusieurs mots en voie d'intégration ou intégrée (...). La lexie complexe est une séquence figée (...) », J. Dubois et al., Dictionnaire de linguistique, Larousse, 1973, pp. 206-207.

7. Selon Le Bon usage (Grévisse, 1969 : p. 81, § 128, note 2), dans « Dérivation nominale » : «le mot nominal est pris dans le sens général et a rapport tant aux adjectifs qu'aux noms proprement dits. Sous l'appellation générique de nomen les grammairiens latins rangeaient le nomen substantivum substantif) et le nomen adjectivum (nom adjectif)». 89 dérivation savante laine : «les suffixes latins employés par a 
formation savante ont produit, par dérivation nominale, des noms et des adjectifs; par dérivation verbale, ils ont forni des verbes.

8. Cf. Paul Bogaards, On ne parle pas français : la langue française face à l'anglais, De Boeck Supérieur, 2008.

\section{RÉSUMÉS}

L'article propose une partie de l'étude analytique de la typologie des procédés néologiques dans le journal francophone algérien, Le Quotidien d'Oran, celle concernant l'affixation. Par le biais de cette analyse, nous tâcherons de relever et de décrire les néologismes les plus significatifs de l'affixation dans les chroniques de ce journal. La typologie des néologismes que nous avons adoptée est celle proposée notamment par J.F Sablayrolles dans La néologie en français contemporain. Examen du concept et analyse de productions néologiques récentes (2000). Nous proposons en annexe (2) un tableau de tous les procédés de la néologie à l'œuvre dans les chroniques de Tranche de vie, de 2009 à 2014 avec des exemples, et qui feront l'objet d'autres publications.

The article proposes a part of an analytical study of the typology of neologisms processes in the Algerian French-language newspaper Le Quotidian d'Oran, which concerns affixation. Through this analysis, we will try to identify and describe the most significant neologisms of affixation in the chronicles of this newspaper. The typology of neologisms that we have adopted is that proposed mainly by JF Sablayrolles especially in La néologie en français contemporain. Examen du concept et analyse de productions néologiques récentes (2000). We propose in the appendix (2) a table of all of the processes at work in the chronicles of "Tranche de vie" from 2009-2014 with examples, which will be subject to other publications.

\section{INDEX}

Mots-clés : néologisme, affixation, suffixation, préfixation, formation parasynthétique, Le Quotidien d'Oran

Keywords : neologism, affixation, suffixation, prefixing, parasynthétique formation, Le Quotidien d'Oran

\section{AUTEUR}

\section{SAMIRA ALLAM-IDDOU}

Université de Mostaganem 